\title{
Comparative safety of BRAF and MEK inhibitors (vemurafenib, dabrafenib and trametinib) in first-line therapy for BRAF-mutated metastatic melanoma
}

\author{
ANA CEBOLLERO, TERESA PUÉRTOLAS, ISABEL PAJARES, LOURDES CALERA and ANTONIO ANTÓN
}

Department of Medical Oncology, University Hospital Miguel Servet, 50009 Zaragoza, Spain

Received April 20, 2016; Accepted June 13, 2016

DOI: $10.3892 / \mathrm{mco} .2016 .978$

\begin{abstract}
A retrospective observational study was conducted on patients diagnosed with serine/threonine-protein kinase B-Raf (BRAF)-mutated metastatic melanoma, who underwent first-line therapy with BRAF and mitogen-activated protein kinase kinase (MEK) inhibitors (vemurafenib, dabrafenib or a combination of dabrafenib and trametinib) at the Miguel Servet University Hospital (Zaragoza, Spain) between November, 2011 and August, 2015. The aim of this study was to analyse the toxicity produced by BRAF and MEK inhibitors. The most common toxicities were similar to those published in clinical trials, particularly arthralgia, alopecia and photosensitivity in the vemurafenib group; asthenia, hyperkeratosis and dry skin in the dabrafenib group; and diarrhoea and dry skin in the dabrafenib plus trametinib group. Toxicities that had not been described in clinical trials were also identified. Thus, the present study confirmed that the results obtained in clinical trials are similar to those obtained in clinical practice.
\end{abstract}

\section{Introduction}

Melanoma is a malignant tumour of melanocytes, which are the cells that produce the pigment melanin and originate from the neural crest. Although the majority of melanomas develop on the skin, they may also occur on mucosal surfaces or other locations to which cells from the neural crest migrate, including the uveal tract.

The incidence of melanoma has been increasing in recent years, with $\sim 160,000$ cases diagnosed annually worldwide (79,000 men and 81,000 women), which represents a percentage of $1.5 \%$ of all tumours in both genders. Melanoma is more common among women in Europe, with the highest incidence recorded in countries with strong solar irradiance

Correspondence to: Dr Ana Cebollero, Deparment of Medical Oncology, University Hospital Miguel Servet, 3-5 Avda Isabel la Católica, 50009 Zaragoza, Spain

E-mail: acebolleromiguel@gmail.com

Key words: melanoma, toxicity, serine/threonine-protein kinase $\mathrm{B}-\mathrm{Raf} / \mathrm{mitogen}$-activated protein kinase kinase inhibitors and non-native white populations, such as Australia, New Zealand, the United States of America and South Africa. In Europe, melanoma is more common in the north and west (populations with very white skin, exposed to the sun particularly during summer). However, while the frequency of melanoma occurrence tends to stabilise and even decrease in all the abovementioned areas, it continues to increase in the southern and eastern parts of Europe (1).

Approximately 3,600 cases are diagnosed annually in Spain. Similar to the rest of Europe, this type of tumour is more common among women compared with men (2.7 vs. $1.5 \%$, respectively). The incidence in Spain may be considered as high (global adjusted rate in 2002: 5.3 new cases/100,000 inhabitants/year in men and 5.5 in women), with a significant increase, particularly since the 1990s.

Cases of melanoma have been recorded at all ages, although the majority are diagnosed between the ages of 40 and 70 years (2).

The survival rates for melanoma depend on the stage at which it is diagnosed. For early-stage disease (IA-IB), the overall survival rate is $92-97 \%$ at 5 years and $86-95 \%$ at 10 years; however, in more advanced stages (IIIA-IIIC), the overall survival rate is $40-78 \%$ at 5 years and $24-68 \%$ at 10 years (3).

The risk factors may be intrinsic (genetic and phenotypic) and extrinsic (environmental and exposure-related). The most significant risk factors are considered to be sun exposure, pigmentation characteristics, the presence of multiple moles, immunodepression and certain types of environmental exposure (4).

Patients presenting with a suspicious pigmented lesion should undergo excisional biopsy, preferably with clear margins.

Patients with stage I-II localised melanomas should complete the diagnosis with a selective sentinel lymph node biopsy (SLNB), as the SLNB status is the most important prognostic factor in patients with localised melanoma (5).

The most important prognostic factors have been incorporated into the revised 2009 American Joint Committee on Cancer staging guidelines (6) and include the following: Thickness and/or level of invasion; mitotic index, defined as mitoses per mm; ulceration or bleeding at the primary site; number of regional lymph nodes involved, with distinction of systemic macrometastasis and micrometastasis; systemic 
metastasis (non-visceral vs. lung vs. all other visceral sites); and elevated serum lactate dehydrogenase (LDH) level $(3,4,7,8)$.

The therapeutic options for melanoma include surgery, immunotherapy, chemotherapy and radiotherapy.

Surgery includes excisional biopsy, with the margin width depending on Breslow thickness (8) (measured in $\mathrm{mm}$ of vertical growth/invasion of the melanoma from the granular layer). For patients with melanomas with a Breslow thickness of $1 \mathrm{~mm}$, the surgical margin is established at $1 \mathrm{~cm}$; for a Breslow thickness of 1-2 mm, the margins should be $1-2 \mathrm{~cm}$; finally, for a Breslow thickness of $>2 \mathrm{~mm}$, a 2-cm margin is recommended. SLNB is performed in patients with a Breslow thickness of $>1 \mathrm{~mm}$ and those with a Breslow thickness of $0.75-1 \mathrm{~mm}$ and certain risk factors, such as tumour ulceration, age $<40$ years, a Clark level of IV, regression, or a high mitotic index (8). Lymph node drainage should be performed if the result of SLNB is positive, or if there are clinically positive nodes (stage IIB or IIIC).

Despite surgical treatment, a significant proportion of patients relapse. The most important factors for relapse include tumour thickness, mitotic index, presence of ulceration and nodal metastasis. High-risk patients, such as those with stage IIB-C (T4 or with ulceration) and stage III disease, are candidates for adjuvant therapy (9).

Adjuvant therapy with high doses of interferon- $\alpha$ has been shown to be beneficial in terms of progression-free survival, but not overall survival, in patients with resected stage IIB-III melanoma $(10,11)$. Pegylated interferon has also shown benefits with regard to relapse-free survival in patients with nodal metastasis (12). The adjuvant therapy options for stage IIB-IIC patients include clinical trial, observation, or high doses of interferon- $\alpha$ (category 2B). Adjuvant therapy options for stage III patients include clinical trial, observation, or high doses of interferon- $\alpha$ (category $2 \mathrm{~B}$ ).

Adjuvant radiotherapy may be considered for selected patients with clinical lymph node metastasis and risk factors predicting a high risk of nodal relapse (category 2B) (13).

Over the last decade, there have been advances in targeted therapies in the field of metastatic melanoma. These therapies have led to a change in therapeutic strategies, as they are proving to be more effective compared with conventional chemotherapy.

In stage III studies, dacarbazine, the only conventional chemotherapy drug approved for the treatment of metastatic melanoma, was associated with a response rate of $7-12 \%$ and a median overall survival of 5.6-7.8 months $(14,15)$. Chemotherapy drug combinations may increase the response rates, but exert no effect on survival.

The use of ipilimumab, a monoclonal antibody that binds to cytotoxic T-lymphocyte-associated protein 4 , has been demonstrated to improve survival when compared to a peptide vaccine (16). In combination with dacarbazine, ipilimumab has been associated with better overall survival when compared to dacarbazine monotherapy (17).

Approximately 40-60\% of metastatic melanoma patients harbour a BRAF mutation, which entails the activation of a signalling cascade through the mitogen-activated protein (MAP) kinase pathway, involved in such cell processes as proliferation, differentiation, survival, stress response and apoptosis $(18,19)$.
Vemurafenib is a powerful inhibitor of mutated BRAF. Dabrafenib is a reversible, ATP-competitive inhibitor that selectively inhibits BRAF kinase.

Both drugs have achieved an increase in the rates of progression-free and overall survival in stage III clinical trials compared with conventional chemotherapy in patients with BRAF-mutated metastatic melanoma $(20,21)$.

However, the majority of these patients develop resistance, which is associated with a median progression-free survival of 6-7 months. The majority of the resistance mechanisms include reactivation of the MAP kinase pathway (22-24). Trametinib, a mitogen-activated protein kinase kinase (MEK) inhibitor, has been associated with an increase in overall survival in patients with BRAF-mutated metastatic melanoma, compared with chemotherapy (25).

It has been observed that the combination of BRAF and MEK inhibitors (dabrafenib and trametinib), compared with BRAF inhibitors as monotherapy, delays the appearance of resistance and reduces the incidence of hyperproliferative skin lesions. This was the reasoning behind a series of stage III clinical trials, one of which demonstrated an increase in overall survival resulting from the combination of BRAF and MEK inhibitors, compared with BRAF inhibitor monotherapy, without a notable increase in toxicity (26).

In a clinical trial comparing vemurafenib to chemotherapy, an increase in progression-free survival was observed in favour of vemurafenib [5.6 vs. 1.6 months; hazard ratio $(\mathrm{HR})=0.26$; $95 \%$ confidence interval (CI): $0.20-0.33$ ], with an overall survival rate at 6 months of $84 \%$ (95\% CI: 78-89) for the vemurafenib group vs. $64 \%$ (95\% CI: 56-73) for the chemotherapy group. The majority of the patients exhibited a reduction in tumour size with vemurafenib, with a $48 \%$ of confirmed objective responses (including 2 patients with complete response and 104 patients with partial response) (20).

In the clinical trial that compared vemurafenib to dabrafenib in combination with trametinib, the progression-free survival for the vemurafenib group was 7.3 months vs. 11.4 months for the combination group $(\mathrm{HR}=0.56 ; 95 \% \mathrm{CI}: 0.46-0.69)$ with an overall survival rate at 12 months of $72 \%$ (95\% CI: 67-77) for the combination group vs. $65 \%$ (95\% CI: 59-70) for the vemurafenib group. The overall survival rate for the vemurafenib group was 17.2 months $(\mathrm{HR}=0.69$; 95\% CI: 0.53-0.89), while overall survival had not been reached by the combination group when the data were published. Of the patients in treatment with the drug combination, $13 \%$ had a complete response vs. $7 \%$ in the vemurafenib group, with $64 \%$ partial responses vs. $52 \%$ in the vemurafenib group.

Clinical guidelines currently recommend treatment with BRAF inhibitors, either as monotherapy or in combination with MEK inhibitors (category 1) for patients with BRAF-mutated metastatic melanoma (9).

The toxicity associated with these types of targeted therapies differs significantly from the toxicity caused by conventional chemotherapy, which makes it crucial to recognise and effectively treat these toxicities.

The main toxicities reported for vemurafenib in clinical trials are cutaneous adverse events (44\%) (rash/keratoacanthoma/hyperkeratosis/skin tags/hand-foot syndrome/cutaneous squamous cell carcinoma), arthralgia $(21 \%)$, fatigue $(13 \%)$, alopecia $(8 \%)$, diarrhoea $(6 \%)$, nausea 
(8\%) and photosensitivity (20). The most commonly described toxicities for dabrafenib are pyrexia (28\%), cutaneous adverse events (32\%) (hyperkeratosis/skin tags/ hand-foot syndrome), asthenia (35\%), arthralgia (27\%) and headache (29\%) (21). The main toxicities described for the dabrafenib-trametinib combination are pyrexia $(51 \%)$, nausea $(30 \%)$, diarrhoea (24\%), chills (30\%), fatigue (35\%), headache (30\%) and vomiting (20\%) (26).

The aims of this study were to analyse the toxicity of therapy with BRAF and MEK inhibitors in clinical practice and to compare the resulting data with those obtained from clinical trials.

\section{Materials and methods}

Patient inclusion criteria and evaluation. A retrospective observational study was conducted on patients diagnosed with BRAF-mutated metastatic melanoma who received first-line therapy with BRAF and/or MEK inhibitors, either as monotherapy or in combination, at the Miguel Servet University Hospital (Zaragoza, Spain) between November, 2011 and August, 2015. The inclusion criteria consisted of a histological diagnosis of unresectable stage IIIC/IV melanoma, presence of a BRAF mutation, age $>18$ years, and no prior treatment with BRAF and/or MEK inhibitors. The exclusion criteria were an Eastern Cooperative Oncology Group (ECOG) performance status score of $>2$, unmeasurable disease and inadequate organ function.

Toxicity was evaluated using the US National Cancer Institute Common Terminology Criteria for Adverse Events, version 4.0 (27).

The variables recorded included date of birth, gender, TNM stage at treatment initiation (M1a, M1b, M1c, unresectable IIIC), Eastern Cooperative Oncology Group (ECOG) performance status score at treatment initiation, presence or absence of brain metastasis, therapy received (vemurafenib, dabrafenib, dabrafenib in combination with trametinib), treatment start/end date, standard dose, dose reduction, described toxicities, degree and time of appearance (immediate, early or late), best response achieved (complete response, partial response or stable disease) according to the Response Evaluation Criteria in Solid Tumours (RECIST) (28), reason for suspension of treatment (disease progression, death, toxicity, patient refusal), administration of subsequent treatment, date of progression and date of death/final follow-up.

Statistical analysis. Clinical data were obtained from patient clinical records in paper form. Statistical analysis was performed using the SPSS Statistics software package. The arithmetic median and standard deviation were used as statistics.

\section{Results}

Patient characteristics. Of the 23 patients analysed, 11 were treated with vemurafenib, 6 with dabrafenib and 6 with a combination of dabrafenib and trametinib, 13 patients were women and 14 patients presented with stage M1c at diagnosis. The median age at treatment initiation was $59 \pm 11.9$ years. The majority of the patients had a good performance status at treatment initiation ( $0 / 1$ in 21 patients). Of the 23 patients, only 4 presented with brain metastasis at treatment initiation. The
LDH levels were over the normal range in at least half of the patients, with a mean value of $287 \pm 249.6$ IU/1.

Treatment. The majority of the patients $(n=20)$ started therapy at a standard dose, compared with 3 who started off at a reduced dose due to associated comorbidities. During the course of treatment, $43.5 \%$ of the patients had to reduce the starting dose by 1 level and $9 \%$ had to reduce the dose by 2 levels (according to the clinical trial criteria). The main reasons for dose reduction were hand-foot syndrome $(8.7 \%)$, low-grade fever $(8.7 \%)$, asthenia $(4.3 \%)$, photosensitivity (4.3\%), hypertransaminasaemia/hyperbilirubinaemia $(4.3 \%)$, prolongation of the QT interval (4.3\%), fluid retention $(4.3 \%)$ and one episode of autoimmune thyroiditis (4.3\%). The median overall treatment duration for all patients was $11 \pm 6.7$ months.

Response to treatment. The treatment response was evaluated by means of RECIST; 3 patients achieved a complete response, 14 achieved a partial response and 5 achieved disease stabilisation.

Of the patients in whom treatment was suspended, $84 \%$ was due to disease progression, whereas only $10 \%$ cases with suspended treatment were attributed to unacceptable toxicity. Of the 23 patients, 18 received treatment subsequent to the inhibitor, mostly with standard chemotherapy.

Toxicity. As regards toxicity, the main adverse effects among patients treated by monotherapy with vemurafenib $(n=11)$ were arthralgia, alopecia, asthenia, photosensitivity, hyperkeratosis, dry skin and fluid retention. Arthralgia and alopecia appeared in all 11 patients $(100 \%)$, photosensitivity in $10(91 \%)$, dry skin in $9(82 \%)$ and asthenia and hyperkeratosis in 8 patients $(73 \%)$. These toxicities were all grade 1-2, except for 1 patient who developed grade 3 photosensitivity, which was the reason for treatment suspension.

Moreover, during treatment with vemurafenib, there was one case of grade 3 prolongation of the QT interval with immediate onset, and one case of grade 1 prolongation of the QT interval with delayed onset. There was one case of basal cell carcinoma, which was surgically removed without subsequent incidents, and one case of grade 3 hand-foot syndrome, which led to a reduction in dosage. Fluid retention appeared in half of the cases, mostly with early onset, and of grade 3 severity in one patient.

There was one case of paralysis of the facial nerve and a case of grade 3 autoimmune thyroiditis with delayed onset, which was the cause of a definitive suspension of treatment.

The side effects suffered by the group of patients treated with dabrafenib monotherapy $(n=6)$ were arthralgia, asthenia, hyperkeratosis, dry skin and curly hair growth. Cutaneous adverse events were more common, with hyperkeratosis, dry skin and facial erythema in 6 patients $(100 \%)$, followed by arthralgia and asthenia in 5 patients (83\%). All these toxicities were grade 1-2, with the onset mostly during the first weeks of treatment. There was also one case of grade 1 vitiligo with immediate onset and one case of hair colour change with delayed onset.

The most severe toxicity was one case of grade 3 hand-foot syndrome with delayed onset, the consequence of which was treatment suspension. 
Finally, in the group of patients treated with dabrafenib in combination with trametinib $(n=6)$, there was less general toxicity compared with the previously described treatments, and also a reduction in the frequency of adverse effects. The most common toxicities were grade 1-2 diarrhoea in 5 patients $(83 \%)$ and grade $1-2$ dry skin in 3 patients $(50 \%)$. Analytical alterations were observed more frequently compared with the other two treatment groups. Of note, there was one case with grade 2 hypertransaminasaemia, which led to the reduction of the dosage by one level; one case with a grade 3 increase in creatine phosphokinase with early onset; one case with grade 1 diminished renal function with delayed onset; and one case with grade 1 hypophosphataemia with delayed onset.

\section{Discussion}

The demographic and clinical characteristics of the patients in the three treatment groups were very similar compared with the clinical trials conducted. The median age of the patients at treatment initiation in the clinical trials was $<65$ years. The majority of the patients had an ECOG performance status of 0 and 1 (our sample included certain patients with an ECOG performance status of 2) and the patients presented with advanced clinical stages, the greatest proportion being stage M1c.

Unlike in the clinical trials, our sample included more patients with LDH levels above the normal range, and patients with brain metastasis, which is associated with a worse prognosis $(20,21)$. In the clinical trials, where patients presented with brain metastasis, these were to have been treated previously, without exhibiting an increase in the size of the lesions over a period of $\leq 12$ weeks prior to recruitment.

The most common reasons for treatment suspension in the clinical trials was pyrexia (3\%) and reduced ejection fraction $(3 \%)$, both in the group of patients treated with dabrafenib monotherapy, and in the group treated with dabrafenib in combination with trametinib (in the two clinical trials that analysed the efficacy of this combination) $(21,26)$. The most common reason for suspending treatment in the vemurafenib group was arthralgia (2\%), while rash was the most common reason for reducing the dosage and interrupting treatment (14 and 11\%, respectively) (20). In the case of dabrafenib, as monotherapy or in combination with trametinib, the most common cause for reducing the dosage and interrupting treatment was pyrexia (30 and 14\%, respectively) $(21,26)$.

In the present study, the reasons for reducing dosage were hand-foot syndrome (9\%) in the vemurafenib group; pyrexia and diarrhoea (16\%) in the dabrafenib in combination with trametinib group; and arthralgia, hand-foot syndrome and pyrexia $(16 \%)$ in the dabrafenib monotherapy group.

Special mention should be made of 2 cases of reduced dosage in our study that led to a definitive suspension of treatment in the vemurafenib monotherapy group, 1 due to an episode of autoimmune thyroiditis, and 1 due to prolongation of the QT interval.

Side effects in the vemurafenib group led to a reduction of the dosage or interruption of treatment in $38-56 \%$ of the patients in clinical trials (20), compared with $54 \%$ in our sample.
Side effects in the dabrafenib plus trametinib group in previous trials $(21,26)$ led to the interruption of treatment in $55 \%$ of the patients, compared with $25 \%$ in our study.

In the clinical trial comparing vemurafenib to dacarbazine (20), arthralgia (21\%), asthenia (13\%) and cutaneous adverse events (rash) (18\%) were the most common side effects in the vemurafenib group. Grade 2-3 photosensitivity appeared in $12 \%$ of the patients evaluated, compared with $91 \%$ in our study. One significant difference was the development of squamous cell carcinoma in several patients in that clinical trial $(12 \%)$, whereas no cases were reported in our sample.

In the clinical trial that compared vemurafenib to dabrafenib combined with trametinib (26) the most common side effects in the vemurafenib group were arthralgia (51\%), rash (43\%), alopecia (39\%), diarrhoea (38\%), nausea (36\%) and photosensitivity $(22 \%)$.

In the clinical trial that compared dabrafenib to dabrafenib combined with trametinib (25) the most common side effects in both groups were pyrexia ( 28 and $51 \%$, respectively), asthenia (35\%), nausea (26 and 30\%, respectively), headache (29 and $30 \%$ respectively), chills (16 and $30 \%$ respectively), arthralgia (27 and 24\% respectively), rash (22 and 23\% respectively) and hypertension (14 and 22\% respectively). The frequency with which these side effects appeared in our study was lower, with the most common toxicities being asthenia and arthralgia (83\%) in the dabrafenib group, and diarrhoea (83\%) and cutaneous adverse events (90\%) in the combination group. Furthermore, no cases of rash or hypertension were reported in either of the two groups (dabrafenib monotherapy and dabrafenib in combination with trametinib). A noteworthy characteristic of the clinical trials is that the combination group (dabrafenib plus trametinib) had fewer cutaneous adverse events compared with the group of patients receiving dabrafenib monotherapy. Of these, mention should be made of the lower incidence of hyperkeratosis, skin tags, alopecia and cutaneous squamous cell carcinoma.

The proportion of patients suffering from grade 3 toxicities was low in both studies, with the majority of toxicities being grade 1-2. No toxicity-related deaths were reported in our study.

Despite the limitations of our study, namely its small sample size and the fact that it is a retrospective observational study, with the consequent limitations in data collection, it offers relevant information on the toxicity of treatment with BRAF and MEK inhibitors in patients diagnosed with metastatic melanoma, as the experience in clinical practice is scarce.

The present study demonstrated that the toxicities associated with antineoplastic therapy with BRAF and MEK inhibitors for patients with BRAF-mutated metastatic melanoma in an unselected population were similar to those published in the clinical trials that led to their approval.

\section{References}

1. Ferlay J, Autier P, Boniol M, Heanue M, Colombet M and Boyle P: Estimates of the cancer incidence and mortality un Europe in 2006. Ann Oncol 18: 581-592, 2007.

2. Sáenz S, Conejo-Mir J and Cayuela A: Melanoma epidemiology in Spain. Actas Dermosifiliogr 96: 411-418, 2005.

3. Balch CM, Gershenwald JE, Soong SJ, Thompson JF, Atkins MB, Byrd DR, Buzaid AC, Cochran AJ, Coit DG, Ding S, et al: Final version of 2009 AJCC melanoma staging and classification. J Clin Oncol 27: 6199-6206, 2009. 
4. Slingluff CI Jr, Flaherty K, Rosenberg SA, et al: Cutaneous melanoma. In: DeVita VT Jr, Lawrence TS, Rosenberg SA (eds) Cancer. Principles and Practice of Oncology. 9th edition. Philadelphia, Pa: Lippincott Williams \& Wilkins: pp1643-1691, 2011.

5. Balch CM, Soong SJ, Gershenwald JE, Thompson JF, Reintgen DS, Cascinelli N, Urist M, McMasters KM, Ross MI, Kirkwood JM, et al: Prognostic factors analysis of 17,600 melanoma patients: Validation of the American joint committe on cancer melanoma staging system. J Clin Oncol 19: 3622-3634, 2001.

6. Balch CM, Gershenwald JE, Soong SJ, Thompson JF, Atkins MB, Byrd DR, Buzaid AC, Cochran AJ, Coit DG, Ding S, et al: Final version of 2009 AJCC melanoma staging and classification. J Clin Oncol 27: 6199-6206, 2009.

7. Balch CM, Soong SJ, Ross MI, Urist MM, Karakousis CP, Temple WJ, Mihm MC, Barnhill RL, Jewell WR, Wanebo HJ and Harrison R: Long-term results of a multi-institutional randomized trial comparing prognostic factors and surgical results for intermediate thickness melanomas (1.0 to $4.0 \mathrm{~mm}$ ) Intergroup Melanoma Surgical Trial. Ann Surg Oncol 7: 87-97, 2000 .

8. Manola J, Atkins M, Ibrahim J and Kirkwood J: Prognostic factors in metastatic melanoma: A pooled analysis of eastern cooperative oncology group trials. J Clin Oncol 18: 3782-3793, 2000.

9. Berrocal A, Arance A, Espinosa E, Castaño AG, Cao MG, Larriba JL, Martín JA, Márquez I, Soria A and Algarra SM: SEOM guidelines for the management of malignant melanoma 2015. Clin Transl Oncol 17: 1030-1035, 2015.

10. Kirwood JM, Strawderman MH, Ernstoff MS, Smith TJ, Borden EC and Blum RH: Interferon alfa-2b adjuvant therapy of high-risk resected cutaneous melanoma: The Eastern Cooperative Oncology Group Trial EST 1684. J Clin Oncol 14: 7-17, 1996.

11. Kirwood JM, Ibrahim JG, Sondak VK, Richards J, Flaherty LE, Ernstoff MS, Smith TJ, Rao U, Steele M and Blum RH: High-and low-dose interferon alfa-2b in high risk melanoma: First analysis of intergroup trial E1690/S9111/C9190. J Clin Oncol 18 2444-2458, 2000.

12. Eggermont AM, Suciu S, Santinami M, Testori A, Kruit WH, Marsden J, Punt CJ, Salès F, Gore M, Mackie R, et al: Adjuvant therapy with pegylated interferón alfa- $2 \mathrm{~b}$ versus observation alone in resected stage III melanoma: Final results of EORCT 18991, a randomised phase III trial. Lancet 372: 117-126, 2008.

13. Burmeister BH, Henderson MA, Ainslie J, Fisher R, Di Iulio J, Smithers BM, Hong A, Shannon K, Scolyer RA, Carruthers S, et al: Adjuvant radiotherapy versus observation alone for patients at risk of lymph-node field relapse after therapeutic lymphadenectomy for melanoma: A randomised trial. Lancet Oncol 13: 589-597, 2012.

14. Chapman PB, Einhorn LH, Meyers ML, Saxman S, Destro AN, Panageas KS, Begg CB, Agarwala SS, Schuchter LM, Ernstoff MS, et al: Phase III multicenter randomized trial of the Dartmouth regimen versus dacarbazine in patients with metastatic melanoma. J Clin Oncol 17: 2745-2751, 1999.

15. Middleton MR, Grob JJ, Aaronson N, Fierlbeck G, Tilgen W, Seiter S, Gore M, Aamdal S, Cebon J, Coates A, et al: Randomized phase III study of temozolomide versus dacarbazine in the treatment of patients with advanced metastatic malignant melanoma. J Clin Oncol 18: 158-166, 2000.
16. Hodi FS, O'Day SJ, McDermott DF, Weber RW, Sosman JA, Haanen JB, Gonzalez R, Robert C, Schadendorf D, Hassel JC, et al: Improved survival with ipilimumab in patients with metastatic melanoma. N Engl J Med 363: 711-723, 2010.

17. Robert C, Thomas L, Bondarenko I, O'Day S, Weber J, Garbe C, Lebbe C, Baurain JF, Testori A, Grob JJ, et al: Ipilimumab plus dacarbazine for previously untreated metastatic melanoma. N Engl J Med 364: 2517-2526, 2011.

18. Davies H, Bignell GR, Cox C, Stephens P, Edkins S, Clegg S, Teague J, Woffendin H, Garnett MJ, Bottomley W, et al: Mutations of the BRAF gene in human cancer. Nature 417: 949-954, 2002

19. Curtin JA, Fridlyand J, Kageshita T, Patel HN, Busam KJ, Kutzner H, Cho KH, Aiba S, Bröcker EB, LeBoit PE, et al: Distinct sets of genetic alterations in melanoma. N Engl J Med 353: 2135-2147, 2005.

20. Chapman PB, Hauschild A, Robert C, Haanen JB, Ascierto P, Larkin J, Dummer R, Garbe C, Testori A, Maio M, et al: Improved survival with vemurafenib in melanoma with BRAF V600E mutation. N Engl J Med 364: 2507-2516, 2011.

21. Hauschild A, Grob JJ, Demidov LV, Jouary T, Gutzmer R, Millward M, Rutkowski P, Blank CU, Miller WH Jr, Kaempgen E, et al: Dabrafenib in BRAF-mutated metastatic melanoma: A multicentre, open-label, phase 3 randomised controlled trial. Lancet 380: 358-365, 2012

22. Rizos H, Menzies AM, Pupo GM, Carlino MS, Fung C, Hyman J, Haydu LE, Mijatov B, Becker TM, Boyd SC, et al: BRAF inhibitor resistance mechanisms in metastatic melanoma: Spectrum and clinical impact. Clin Cancer Res 20: 1965-1977, 2014.

23. Van Allen EM, Wagle N, Sucker A, Treacy DJ, Johannessen CM, Goetz EM, Place CS, Taylor-Weiner A, Whittaker S, Kryukov GV, et al: The genetic landscape of clinical resistance to RAF inhibition in metastatic melanoma. Cancer Discov 4: 94-109, 2014.

24. Shi H, Hugo W, Kong X, Hong A, Koya RC, Moriceau G, Chodon T, Guo R, Johnson DB, Dahlman KB, et al: Acquired resistance and clonal evolution in melanoma during BRAF inhibitor therapy. Cancer Discov 4: 80-93, 2014.

25. Flaherty KT, Robert C, Hersey P, Nathan P, Garbe C, Milhem M, Demidov LV, Hassel JC, Rutkowski P, Mohr P, et al: Improved survival with MEK inhibition in BRAF-mutated melanoma. N Engl J Med 367: 107-114, 2012.

26. Robert C, Karaszewska B, Schachter J, Rutkowski P, Mackiewicz A, Stroiakovski D, Lichinitser M, Dummer R, Grange F, Mortier L, et al: Improved overall survival in melanoma with combined dabrafenib and trametinib. N Engl J Med 372: 30-39, 2015

27. Dueck AC, Mendoza TR, Mitchell SA, Reeve BB, Castro KM, Rogak LJ, Atkinson TM, Bennett AV, Denicoff AM, O'Mara AM, et al: Validity and Reliability of the US National Cancer Institute's Patient-Reported Outcomes Version of the Common Terminology Criteria for Adverse Events (PRO-CTCAE). JAMA Oncol 1: 1051-1059, 2015.

28. Eisenhauer EA, Therasse P, Bogaerts J, Schwartz LH, Sargent D, Ford R, Dancey J, Arbuck S, Gwyther S, Mooney M, et al: New response evaluation criteria in solid tumours: Revised RECIST guideline (version 1.1). Eur J Cancer 45: 228-247, 2009. 